\title{
Maternal tobacco smoke increased visceral adiposity and serum corticosterone levels in adult male rat offspring
}

\author{
Erin K. Zinkhan'1, Brook Y. Lang' ${ }^{1}$ Baifeng Yu' ${ }^{1}$ Yan Wang ${ }^{1}$, Chengshe Jiang ${ }^{1}$, Melanie Fitzhugh ${ }^{1}$, Marjanna Dahl',
} Michael S. Campbell', Camille Fung', Daniel Malleske' ', Kurt H. Albertine' ', Lisa Joss-Moore' and Robert H. Lane ${ }^{2}$

BACKGROUND: Maternal tobacco smoke (MTS) predisposes human and rat offspring to visceral obesity in early adulthood. Glucocorticoid excess also causes visceral obesity. We hypothesized that in utero MTS would increase visceral adiposity and alter the glucocorticoid pathway in young adult rats.

METHODS: We developed a novel model of in utero MTS exposure in pregnant rats by exposing them to cigarette smoke from E11.5 to term. Neonatal rats were cross-fostered to control dams and weaned to standard rat chow through young adulthood (postnatal day 60).

RESULTS: We demonstrated increased visceral adiposity $(193 \%)^{*}$, increased visceral adipose 11- $\beta$ hydroxysteroid dehydrogenase 1 mRNA (204\%)*, increased serum corticosterone $(147 \%)^{*}$, and no change in glucocorticoid receptor protein in adult male MTS rat offspring. Female rats exposed to MTS in utero demonstrated no change in visceral or subcutaneous adiposity, decreased serum corticosterone (60\%)*, and decreased adipose glucocorticoid receptor protein $(66 \%)^{*}$. ${ }^{*} P<0.05$.

CONCLUSION: We conclude that in utero MTS exposure increased visceral adiposity and altered in the glucocorticoid pathway in a sex-specific manner. We speculate that in utero MTS exposure programs adipose dysfunction in adult male rat offspring via alteration in the glucocorticoid pathway.

M aternal tobacco smoke (MTS) impacts more than 13\% of pregnancies despite aggressive antismoking campaigns (1). In adult human studies, MTS exposure in utero predisposes to the development of metabolic syndrome, including increased visceral adiposity (2). The increased adiposity seen after gestating in an adverse intrauterine environment, such as maternal tobacco or nicotine exposure, is often adipose depot specific, with the visceral adipose tissue (VAT) increasing in excess to the subcutaneous adipose tissue (SAT) $(3,4)$.

VAT seems to have a detrimental role in the development of metabolic syndrome $(5,6)$. Even when considering dietary and lifestyle choices, MTS exposure in utero independently increases the risk of developing visceral obesity $(7,8)$. Children whose mothers smoked during pregnancy had decreased birth weights yet an increased obesity and BMI index as early as 9-10 $y$ of age, independent of dietary intake and activity levels (8). Furthermore, the effect of MTS on body weight was stronger when mothers smoked during pregnancy compared with that of isolated smoke exposure in childhood (7). Taken together, these findings suggest that MTS exposure during this developmentally sensitive time period both causes intrauterine growth restriction (IUGR) and adversely programs obesity later in life.

Little insight exists on how MTS programs increased visceral adiposity. Rodent studies examining the effect of in utero MTS exposure on the offspring have not examined the etiology of increased visceral adiposity. Rodent studies of tobacco smoke exposure only during lactation demonstrate increased adiposity (9). Furthermore, rodent studies of maternal nicotine, one of the many compounds found in tobacco smoke, demonstrate increased obesity in adult offspring when exposed during gestation or lactation (10-12). One potential mechanism through which MTS and maternal nicotine may program increased visceral adiposity includes alteration of glucocorticoid action on adipocytes. One function of glucocorticoids is to increase fat deposition in the body. Syndromes that increase glucocorticoid levels demonstrate increased visceral adiposity. Tissue response to glucocorticoids involves both depot-specific changes in levels of glucocorticoid receptors (GRs) and local formation of active glucocorticoids. Glucocorticoid signaling occurs through activation of the GR variants, including GR- $\alpha$, which induce transcription of an array of proadipogenic factors. The conversion of inactive glucocorticoid (cortisone in humans, 11-dihydrocorticosterone in rodents) to active glucocorticoid (cortisol in humans, corticosterone in rodents) is catalyzed by the adipocyte enzyme 11- $\beta$ hydroxysteroid dehydrogenase 1 (11- $\beta$ HSD 1). Thus, determination of relative amounts of $11-\beta$ HSD 1 and GR in adipose depots may provide insight into the mechanism through which MTS programs increased visceral adiposity. However, despite the importance of MTS programming increasing visceral adiposity in the offspring, to date relative amounts of GR and 11- $\beta$ HSD 1 in rats exposed to MTS in utero have not been studied.

Using a novel model of MTS exposure during pregnancy, we hypothesized that in utero MTS exposure would program 
adipose tissue dysregulation in young adult rat offspring. Specifically, MTS would increase visceral adiposity, serum corticosterone levels, and VAT GR and 11- $\beta$ HSD 1 levels.

\section{RESULTS}

\section{Weight Gain, Food Intake, and Cotinine Levels}

No MTS-exposed dams died nor had to be removed from MTS exposure because of illness during these experiments. MTSexposed rat dams gained less weight and ate less food during smoke exposure compared with control dams (Table 1). Litter sizes and pup male-to-female ratios were same in all groups. MTS increased maternal serum cotinine levels compared with control dams. Correspondingly, MTS increased fetal serum cotinine levels compared with control fetuses (not detected in the control neonatal rats, and $306 \pm 18 \mathrm{ng} / \mathrm{ml}$ in the neonatal MTS rats, $P<0.05$, pooled serum from the whole litter).

All MTS-exposed pups weighed 12\% less than their gendermatched control pups at birth (Table 2). In addition, placentae from MTS-exposed rat dams weighed 6\% less compared with control placentae. By postnatal day 21, male and female MTS rats continue to weigh less than control male and female rats, respectively (MTS male: $57.3 \mathrm{~g}$, control male: $60.9 \mathrm{~g}, P<0.001$; MTS female $55.4 \mathrm{~g}$, control female: $59.3 \mathrm{~g}, P<0.001$ ). However, by postnatal day 60 , MTS males and females caught up to the body weight of respective control rats. MTS decreased food and caloric intake in males at postnatal day 60 .

Table 1. Effect of tobacco smoke exposure on pregnant dams

\begin{tabular}{lcc}
\hline & $\begin{array}{c}\text { Controls } \\
(n=28 \text { dams })\end{array}$ & $\begin{array}{c}\text { MTS } \\
(n=32 \text { dams })\end{array}$ \\
\hline $\begin{array}{l}\text { Maternal weight gain } \\
\text { (E11-E21) (g) }\end{array}$ & $122.3 \pm 10.4$ & $45.3 \pm 17.3^{*}$ \\
$\begin{array}{l}\text { Maternal food intake } \\
\text { (E11-E21) (g/d) }\end{array}$ & $26.2 \pm 7.7$ & $19.2 \pm 4.8^{*}$ \\
Litter sizes & $11.6 \pm 2.6$ & $10.3 \pm 2.1$ \\
Litter male-to-female ratio & $1: 0.94$ & $1: 0.96$ \\
$\begin{array}{l}\text { Maternal serum cotinine } \\
\text { (ng/ml) }\end{array}$ & Not detected & $119 \pm 27^{*}$ \\
\hline
\end{tabular}

Tobacco smoke exposure decreased maternal weight gain and food intake. MTS increased maternal cotinine levels. Twenty-eight to thirty-two rat dams were analyzed per tobacco smoke exposure group; data represented as mean \pm SEM. MTS, maternal tobacco smoke.

${ }^{*} P \leq 0.05$ MTS dam compared with control dam.

\section{Adipose Depot Quantification, Adipocyte Size, and Total Adipocyte Number}

MTS increased VAT and the ratio of VAT to SAT in males compared with control males (Figure 1). MTS did not alter female VAT, SAT, or ratio of VAT:SAT.

MTS did not change adipocyte size in either VAT or SAT in males or in females in the representative subset VAT and SAT depots (Figure 2). MTS did not change the number of adipocytes per tissue points in either VAT or SAT in males or in females.

\section{Serum Corticosterone and Adipokines}

MTS increased serum corticosterone in males but decreased serum corticosterone in females (Figure 3). MTS did not change serum leptin or adiponectin levels in either sex.

\section{VAT and SAT mRNA and Protein}

With respect to VAT, MTS did not change leptin or adiponectin mRNA in either gender (Figure 4). MTS increased 11- $\beta$ HSD 1 mRNA in males and females. MTS decreased total GR and GR $\alpha$ protein in females, with no change in males (Figure 5).

With respect to SAT, MTS increased leptin and 11- $\beta$ HSD 1 mRNA in males, and adiponectin, and 11- $\beta$ HSD 1 mRNA in females (Figure 4). MTS decreased GR $\alpha$ protein in females, with no change in males (Figure 5).

\section{DISCUSSION}

The primary finding of this study is that MTS exposure in utero increased visceral adiposity and serum corticosterone but not GR protein levels in the adult male rat. Moreover, increased visceral adiposity in the adult male MTS rat occurred despite decreased food intake. Increased visceral adiposity and increased serum corticosterone without a change in VAT GR protein levels in male rats suggest that increased visceral adiposity may be a result of increased corticosterone.

In humans, multiple studies demonstrate that maternal smoking decreases birth weight $(13,14)$. Greater variability in humans exposed to MTS compared with other forms of IUGR may be found from the variation in duration and quantity of tobacco smoke or nicotine intake during gestation. The mechanism through which maternal smoking induces growth restriction is unknown, although likely occurs through multiple mechanisms. These potential mechanisms include

Table 2. Effect of maternal tobacco smoke exposure on offspring

\begin{tabular}{|c|c|c|c|c|}
\hline & Control male & MTS male & Control female & MTS female \\
\hline Body weight at birth (g) ( $n=94-258)$ & $4.26 \pm 0.446$ & $3.77 \pm 0.400^{*}$ & $4.07 \pm 0.409$ & $3.57 \pm 0.448$ \\
\hline Placental weight (g) $(n=64-170)$ & $0.609 \pm 0.111$ & $0.566 \pm 0.110^{*}$ & $0.580 \pm 0.093$ & $0.544 \pm 0.129 *$ \\
\hline Body weight at day $60(\mathrm{~g})(n=12)$ & $373.8 \pm 31.4$ & $366.1 \pm 26.0$ & $230.8 \pm 16.7$ & $225.6 \pm 18.1$ \\
\hline kcal consumed/day $(n=8)$ & $91 \pm 2$ & $76 \pm 3^{*}$ & $44 \pm 3$ & $46 \pm 3$ \\
\hline
\end{tabular}

MTS decreased fetal and placental weights at birth compared with sex-matched controls. MTS males and females caught up to their respective controls by postnatal day 60 . MTS males consumed less food compared with control males. The number of rats per sex per group analyzed is represented above; data represented as mean \pm SEM. 


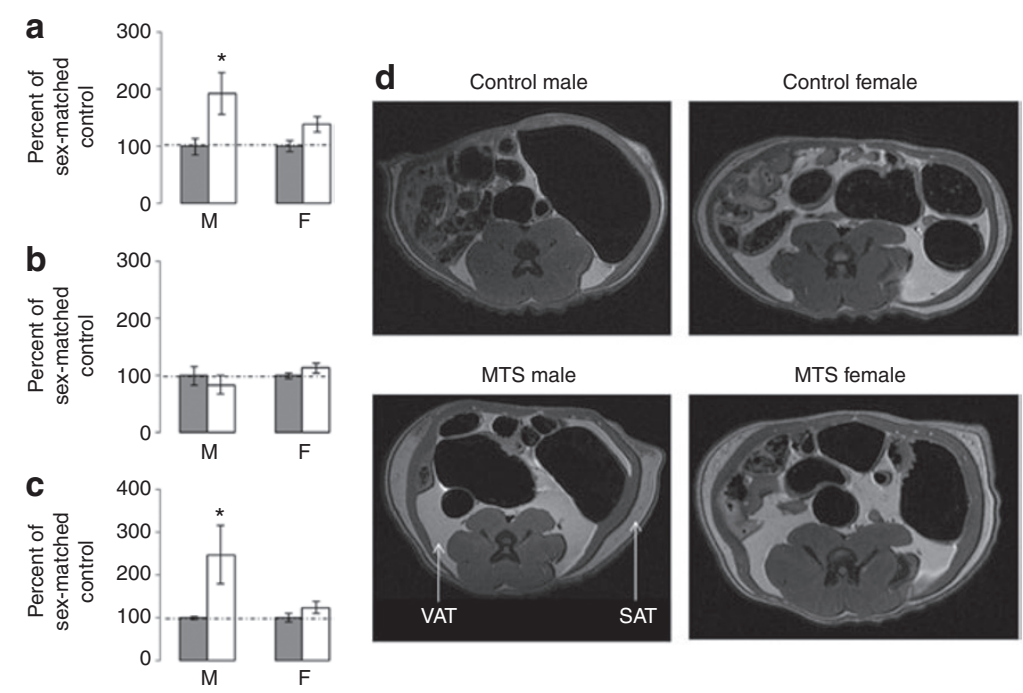

Figure 1. (a) Maternal tobacco smoke (MTS) increased visceral adiposity in male rats. (b) MTS did not change subcutaneous adiposity. (c) However, MTS increased the ratio of VAT to SAT in young adult males. (d) Magnetic resonance images of representative adipose depots in male and female control and MTS rats. $n=4$, data represented as mean \pm SEM. Control data are shown in gray bars, and MTS data in white bars. ${ }^{*} P \leq 0.05$ MTS compared with sexmatched control. SAT, subcutaneous adipose tissue; VAT, visceral adipose tissue.

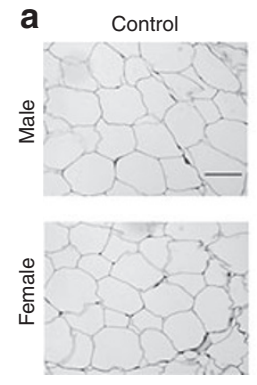

C

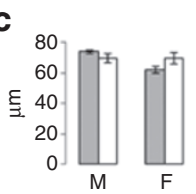

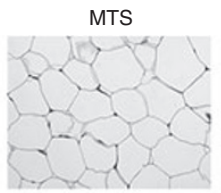

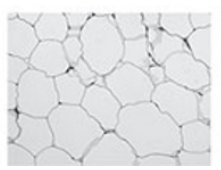

$\mathbf{d}_{80}$

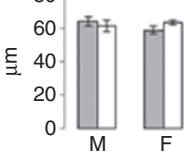

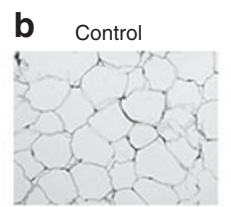
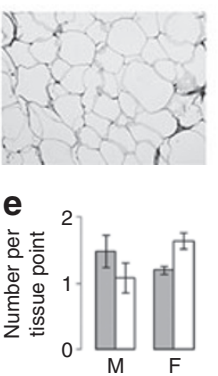

MTS
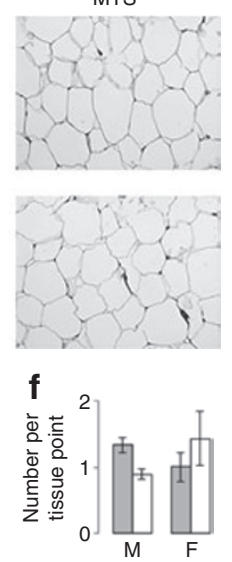

Figure 2. Representative adipose depot images for (a) visceral adipose tissue (VAT) and (b) subcutaneous adipose tissue (SAT); scale bar in the upper left panel of a represents $50 \mu \mathrm{m}$. MTS did not alter adipocyte size in (c) VAT or (d) SAT or adipocyte number per tissue point in (e) VAT or (f) SAT. $n=4$, data represented as mean \pm SEM. Control data are shown in gray bars, and MTS data in white bars. MTS, maternal tobacco smoke.

carbon monoxide and catecholamine-induced vasoconstriction, hypoxia, toxins from the large array of chemicals in tobacco smoke, and decreased nutrient consumption $(15,16)$. Furthermore, unlike other forms of IUGR, MTS exposes the fetus to greater than 4,800 chemicals found in cigarette smoke during a time of sensitivity to developmental programming. One such chemical is nicotine, which induces vasoconstriction, decreasing blood supply to the uterus and developing fetus. The standard measurement used for quantifying nicotine levels is cotinine, a stable metabolite of nicotine. Neonatal rat cotinine levels fell well within the range of 200 to $800 \mathrm{ng} / \mathrm{ml}$, indicating an "active smoker" according to ARUP laboratories.

Not only does MTS predispose the fetus to IUGR, but it also increases the risk of obesity in childhood $(2,7,8,17,18)$. Certainly, social factors interplay with MTS exposure during pregnancy, such as decreased physical activity, parental education, and socioeconomic status associated with postnatal cigarette exposure. Furthermore, catch-up growth, or growth that allows an IUGR fetus to "catch up" in weight to a normally grown peer, may also contribute to the formation of obesity in childhood. Low birth weight humans who had subsequent catch-up growth demonstrated an increased incidence of obesity (19). Similar results have been seen in animal studies. Several rat studies of IUGR induced by nutrient restriction and subsequently allowed ad libitum access to nursing and rat chow showed evidence of increased adiposity $(20,21)$. Similarly, rats in this study demonstrate catch-up growth and visceral adiposity by postnatal day 60 . While catch-up growth likely impacts the results of increased adiposity seen in this study as well as many other human and animal studies, catch-up growth is necessary for proper neurodevelopmental function and thus critical for the IUGR offspring. Furthermore, when correcting 
a

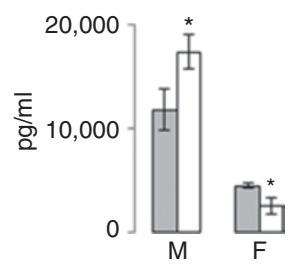

b

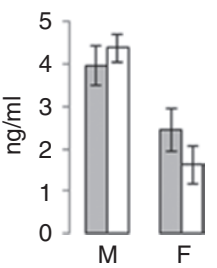

C

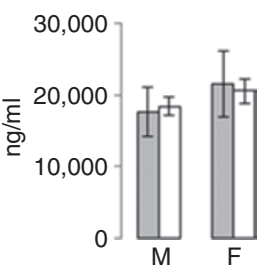

Figure 3. Maternal tobacco smoke (MTS) increased serum corticosterone levels in males (a). MTS decreased serum corticosterone levels in females (a). MTS did not change serum leptin (b) or adiponectin levels (c) in males or females compared with sex-matched controls. $n=6$, data represented as mean \pm SEM. Control data are shown in gray bars, and MTS data in white bars. ${ }^{*} P \leq 0.05$ MTS compared with sex-matched control.

for these factors, MTS remains an independent risk factor for childhood obesity $(2,7,8,17,18)$. The independent risk of obesity with maternal smoking suggests that MTS programs altered adipocyte physiology, leading to increased adiposity in the progeny.

One way through which MTS may increase childhood obesity is through increased caloric consumption in the offspring. In humans, MTS increased highly palatable food consumption and BMI by age $14 \mathrm{y}$ (22). Limited information is available in animal models about caloric consumption in the offspring exposed to MTS in utero, however, maternal nicotine exposure leading to increased caloric consumption has been examined. Prenatal intravenous nicotine exposure in pregnant SpragueDawley rats increased sucrose seeking behavior in offspring without altering locomotor activity (23). Interestingly, in our study of MTS exposure, male offspring consumed fewer total calories as tobacco-naive rats. Increased caloric consumption seen in other studies may be programmed by increased consumption of highly palatable food, but not necessarily typical rat chow. Importantly, MTS increased visceral adiposity despite decreased food intake in adult male rats, suggesting that in utero MTS exposure alters VAT energy storage homeostasis.

Examination of adipose tissue in obese humans demonstrates altered expression of genes involved in glucocorticoid metabolism, such as $11-\beta$ HSD 1 , the enzyme that converts inactive to active glucocorticoid. In obese humans, 11- $\beta$ HSD 1 mRNA and its activity are increased in SAT (24). Furthermore, increased 11- $\beta$ HSD 1 in adipose tissue predicts increased adiposity 2.5 y later (25). Animal models of increased adiposity have shown similar findings. Animals with increased adiposity have increased adipose tissue 11- $\beta$ HSD 1 (26). Importantly, increased 11- $\beta$ HSD 1 seems to be causal for increased visceral adiposity. Transgenic mice that overexpress 11- $\beta$ HSD 1 develop increased visceral adiposity (27) while 11- $\beta$ HSD 1 knockout mice are resistant to developing increased adiposity when fed a high-fat diet (28). Increased 11- $\beta$ HSD 1 in VAT suggests increased glucocorticoid reactivation within the VAT tissue itself, which can lead to increased serum corticosterone as seen in our study. There is also evidence that perinatal stress programs increased adipose tissue 11- $\beta$ HSD $1 \mathrm{mRNA}$ and subsequent increased adiposity in the marmoset offspring (29). Furthermore, maternal nicotine during lactation increases

a

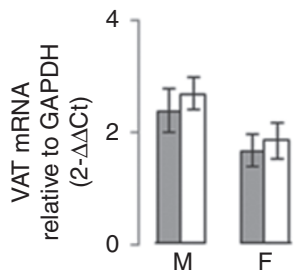

d

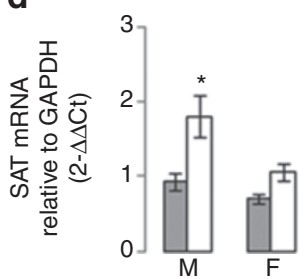

b

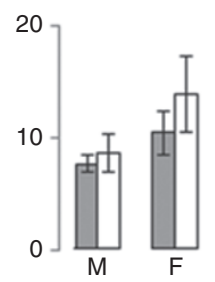

e

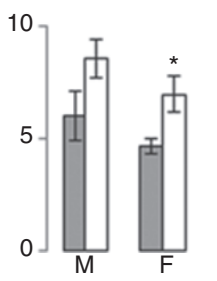

C

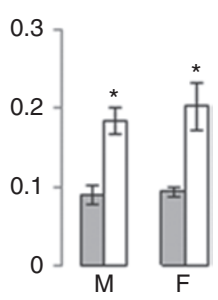

f

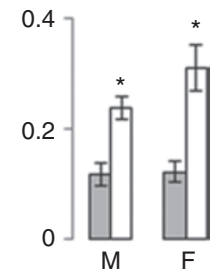

Figure 4. In visceral adipose tissue, maternal tobacco smoke (MTS) did not change leptin (a) or adiponectin mRNA levels (b), but increased 11- $\beta$ hydroxysteroid dehydrogenase 1 (11- $\beta$ HSD 1) mRNA in both males and females (c). In subcutaneous adipose tissue, MTS increased leptin (d) and 11- $\beta$ HSD 1 mRNA in males (f), and adiponectin (e) and 11- $\beta$ HSD 1 mRNA (f) in females. $n=6$, data represented as mean \pm SEM. Control data are shown in gray bars, and MTS data in white bars. ${ }^{*} P \leq 0.05$ MTS compared with sex-matched control. GAPDH, glyceraldehyde-3 phosphate dehydrogenase; SAT, subcutaneous adipose tissue; VAT, visceral adipose tissue. a
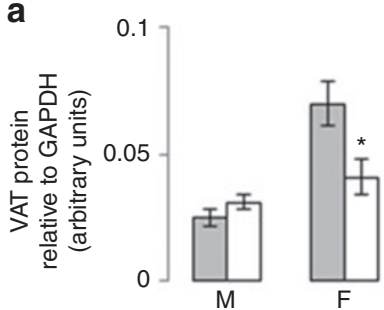

GR

$\beta$-Actin

C

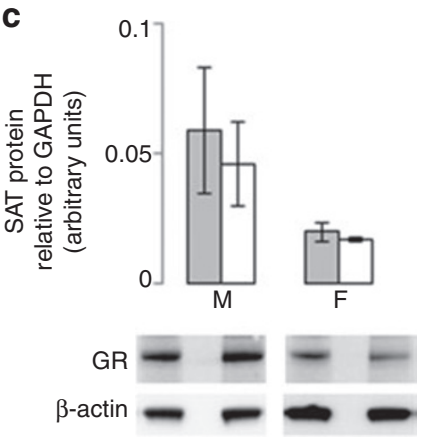

b
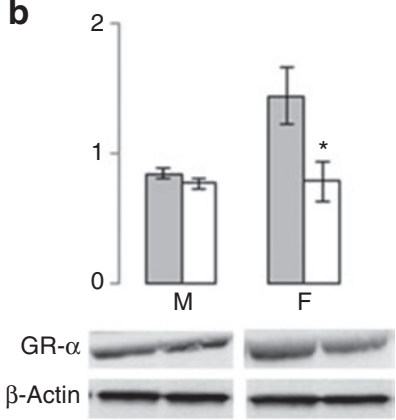

d

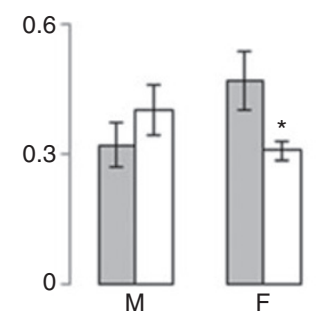

Figure 5. Maternal tobacco smoke (MTS) exposure decreased total GR protein (a) and GR- $\alpha$ protein (b) in female VAT. MTS did not change GR or GR- $\alpha$ protein in either adipose depot in males (a,b). MTS did not change either GR or GR- $\alpha$ protein in SAT in either sex (c). MTS decreased GR- $\alpha$ protein in females in SAT (d). Representative western blots of GR and GR- $\alpha$ relative to control gene $\beta$-actin are shown below the graphical representation of the data. $n=6$, data represented as mean \pm SEM. Control data are shown in gray bars, and MTS data in white bars. ${ }^{*} P \leq 0.05$ MTS compared with sex-matched control. GAPDH, glyceraldehyde-3 phosphate dehydrogenase; GR, glucocorticoid receptor; SAT, subcutaneous adipose tissue; VAT, visceral adipose tissue. 
male offspring adiposity and serum corticosterone levels in rats (30). MTS, another type of perinatal stress, may program increased 11- $\beta$ HSD 1, providing local tissue level and serum increases in glucocorticoid levels and leading to increased visceral adiposity.

Increased visceral adiposity and unchanged visceral adipocyte size indicate that the VAT depot increased by increasing the total number of adipocytes. In animal models of chronic glucocorticoid exposure, chronic glucocorticoid exposure increased the total number of adipocytes in VAT when the whole VAT depot was examined (31). However, chronic glucocorticoid exposure did not change adipocyte size and decreased VAT GR in male rats with increased adiposity (31). Interestingly, despite increased total VAT in MTS males on magnetic resonance imaging, MTS did not change adipocyte size or number of adipocytes in the small subset of representative adipose depot sections. This finding suggests that MTS programs increased the total number of adipocytes throughout the whole VAT depot, but not adipocyte size in the male rat offspring.

Adverse intrauterine environments, such as maternal smoking, often alter metabolic outcomes in a sex-specific manner. In humans, MTS exposure increases visceral adiposity in males earlier than in females $(32,33)$. Animal studies also demonstrate sex specificity in metabolic outcomes from adverse intrauterine environments. Perinatal insults, including MTS, often affect male offspring earlier than female offspring (34-36). Consistent with these findings, male MTS rats demonstrated increased VAT in our study, while female VAT remained unaffected. MTS increased 11- $\beta$ HSD 1 mRNA in both sexes. Consistent with increased 11- $\beta$ HSD 1 mRNA, MTS male rats had increased serum glucocorticoid levels without changes in GR protein levels. MTS may disrupt the mechanisms regulating 11- $\beta$ HSD 1 and GR expression in male rats, and may represent one mechanism through which MTS leads to the formation of increased adiposity in males. Conversely, MTS decreased serum corticosterone and adipose GRs in females. Elevated 11- $\beta$ HSD 1 mRNA can increase local tissue corticosterone without affecting systemic circulating levels (27). Irrespective of the local adipose tissue corticosterone levels, the response to adipose tissue corticosterone may be blunted by decreased adipose tissue GRs in MTS females.

A limitation of this study is that pregnant dams are exposed to tobacco smoke only during the latter half of gestation. This timeframe was chosen because the detrimental health effects of MTS on offspring are largely seen in the second and third trimesters $(8,37)$. Furthermore, this MTS regimen allowed us to ask a specific perinatal question of how prenatal MTS increases visceral adiposity in the adult offspring $(8,37)$. Second, the neonatal MTS rats were cross-fostered to tobacco-naive mothers for rearing consistency. Therefore, neonatal rats were not exposed to postnatal tobacco smoke as many human babies born to tobacco-smoking mothers would be. While we cannot exclude the possibility of cross-fostering affecting the results, we were able to isolate the effects of MTS exposure in utero from extrauterine exposure, which provides valuable insight into a potential mechanism of increased visceral adiposity.
In conclusion, MTS exposure programs adipose dysfunction in adult male rats. Specifically, MTS programs increased visceral adiposity, visceral adipose 11- $\beta$ HSD 1 mRNA, and serum glucocorticoids without changing GR protein in male rats. These findings suggest that MTS adversely programs sexspecific glucocorticoid signaling in VAT of adult rats.

\section{METHODS}

\section{Animal Model}

All animal procedures were carried out according to the US National Institutes of Health (Bethesda, MD) (NIH) Guide for the Care and Use of Laboratory Animals, and the University of Utah Animal Care Committee approved all procedures. Timed pregnant female Sprague-Dawley rats were obtained from Charles River (Wilmington, MA). Rats were exposed to 80 University of Kentucky 3R4F Research Reference Cigarettes (Lexington, KY) per day in a TE-10 Smoke Exposure System (Teague Enterprises, Davis, CA). Rats were exposed to both mainstream and side stream cigarette smoke in four sessions of $35 \mathrm{~min}$ cigarette smoke followed by $35 \mathrm{~min}$ fresh air. Mainstream cigarette smoke was obtained by pulling air through the cigarette. Side stream cigarette smoke was obtained by collecting smoke off the end of the burning cigarette. Providing both mainstream and side stream cigarette smoke to the pregnant dams was chosen because human mothers who smoke cigarettes breathe both mainstream and side stream cigarette smoke. Rats were exposed daily Monday through Friday from E11.5 to term (E21.5) (38). This gestation range was selected because birth weight is most significantly decreased by smoke exposure in the latter half of gestation (8). Tobacco-naive control rats were housed similarly but were not exposed to tobacco smoke. At delivery, all pups were cross-fostered to control dams to isolate prenatal MTS exposure. Litters were culled to six for rearing consistency. Pups were weaned on postnatal day 21, the standard time for weaning, and allowed ad libitum access to food and water for the remainder of the study. Food was weighed weekly for the latter $2 \mathrm{wk}$ of the study ( $2 \mathrm{wk}$ before postnatal day 60 ) to determine food intake. Postnatal day 60 was examined because this time point represents a pubertal or young adult rat, and puberty in the MTS-exposed human is associated with the first signs of obesity (8). A total of 46 MTS-exposed pregnant dams, 42 control dams, and offspring from these dams were used in this study. There were no differences in body weight between subgroups used for the following analyses and the population as a whole.

\section{Measurement of Serum Cotinine Levels}

Cotinine, a stable metabolite of nicotine, was measured to determine serum levels of nicotine. Maternal and fetal serum cotinine levels were measured using a solid phase competitive enzyme-linked immunosorbent assay kit (BQ Kits, San Diego, CA) following manufacturer's protocol. A total of 32 pregnant dams exposed to MTS and 28 pregnant dams as controls were used for measuring maternal cotinine levels, and their respective pooled litters obtained via Cesarean section at E21.5 were used for measuring fetal cotinine levels.

Abdominal Magnetic Resonance Imaging for Fat Quantification Abdominal magnetic resonance images were performed, and data were analyzed as previously described (39). In brief, magnetic resonance imaging was performed on 60-day-old rats at the small animal imaging facility at the University of Utah. Paired images of water and fat plus water were obtained from diaphragm to pelvis. Images were analyzed using ImageJ software (National Institutes of Health, Bethesda, MA). Magnetic resonance images were analyzed from kidney to pelvis for visceral fat, using retroperitoneal fat as a representative visceral fat depot, and from diaphragm to pelvis for subcutaneous fat. Total subcutaneous and visceral fat depots were analyzed for four unrelated rats per sex per group, and these rats were not used for other analyses in this study.

\section{Adipocyte Size and Number}

A small subset of left retroperitoneal adipose was used as a representative VAT depot, and a small subset left flank adipose was used as a 
representative SAT depot. Adipose tissue was fixed in $10 \%$ formalin, embedded in paraffin, sliced to $5 \mu \mathrm{m}$ thick, and stained with hemotoxylin and eosin at the University of Utah Dermatopathology laboratory. Adipocyte diameter was quantified using Bioquant True Color Windows Image Analysis System (R\&M Biometrics, Nashville, TN) measuring five adipocytes per high powered field for 15 high powered fields per adipose depot per rat. Adipocyte number was determined using Bioquant by counting all adipocytes in a high powered field for 15 high powered fields per adipose depot per rat. Adipocyte size and adipocyte number were analyzed from four unrelated rats per sex per group, and these rats were not used for other analyses in this study.

\section{Serum Corticosterone and Adipokine Quantification}

Mixed arterial and venous serum was collected at necropsy after a 6-h fast in serum separator tubes (BD Vacutainer, BD, Franklin Lakes, NJ) and placed on ice. Serum was separated from cellular components within $30 \mathrm{~min}$ of collection, and serum aliquots were frozen at -80 ${ }^{\circ} \mathrm{C}$. Serum corticosterone was quantified using an ELISA kit (Cayman Chemical Company, Ann Arbor, MI), with a kit detection limit of 30 $\mathrm{pg} / \mathrm{ml}$. Serum adiponectin and leptin were quantified using an ELISA kit (Millipore, St Charles, MI), with kit detection limits of $0.08 \mathrm{ng} / \mathrm{ml}$ for leptin and $0.155 \mathrm{ng} / \mathrm{ml}$ for adiponectin. Serum from six unrelated rats per sex per group was analyzed, and these rats were used for adipose tissue mRNA and protein studies.

\section{Adipose Tissue mRNA Quantification}

VAT was obtained from the retroperitoneum as a representative VAT depot. SAT was obtained from the flank. Visceral and SAT was flash frozen in liquid nitrogen and stored at $-80{ }^{\circ} \mathrm{C}$. Total RNA was extracted as described previously using the RNeasy Lipid Tissue Mini Kit (Qiagen, Valencia, CA) for visceral and subcutaneous fat (39). Total RNA was quantified spectrophotometrically. The cDNA was synthesized from $1 \mu \mathrm{g}$ RNA using the High Capacity cDNA Reverse Transcription Kit (Applied Biosystems, Foster City, CA) per manufacturer's protocol.

\section{Real-Time Reverse Transcriptase PCR}

Semiquantitative real-time reverse transcription-PCR quantification was performed using glyceraldehyde- 3 phosphate dehydrogenase as an internal control because Ct values of glyceraldehyde- 3 phosphate dehydrogenase did not differ between control and MTS animals. Relative quantification of PCR products was based on differences between glyceraldehyde-3 phosphate dehydrogenase and the target using the comparative Ct method (TaqMan Gold RT-PCR manual; PE Biosystems, Foster City, CA) (40). Assays were performed with Taqman Gene Expression Assays (Applied Biosystems, Carlsbad, CA) for 11- $\beta$ HSD 1, leptin, and adiponectin.

\section{Adipose Tissue Protein Quantification}

Visceral and SAT protein was isolated using whole-cell lysates in a buffer containing $150 \mathrm{mM} \mathrm{NaCl}, 50 \mathrm{mM}$ Tris $\mathrm{pH} 7.4,1 \mathrm{mM}$ EDTA, $0.25 \%$ Na-deoxycholate, $1 \%$ Igepal CA-630. Total protein was quantified using a bovine serum albumin standard curve. Fifty micrograms protein was run on a $4-12 \%$ sodium dodecyl sulfate-polyacrylamide gel electrophoresis (Bio-Rad, Hercules, CA). Protein was transferred to a polyvinylidene diflouride membrane. Membranes were blocked with $5 \%$ milk. GR and GR- $\alpha$ (ab3578 and ab3580, Abcam, Cambridge, UK) antibodies were used to identify and quantify specific protein content. $\beta$-Actin (A1978, Sigma-Aldrich, St Louis, MO) was used as a loading control. Protein was detected with Western Lightning enhanced chemiluminescence (PerkinElmer Life Sciences, Waltham, MA) using goat anti-rabbit horseradish peroxidase secondary antibody from Cell Signaling Technology. Blots were quantified using a Kodak Image Station 2000R (Eastman Kodak/SIS, Rochester, NY).

\section{Statistics}

Tables and figures were expressed as mean \pm SEM. Data analysis was completed with ANOVA (Fisher's protected least-significant difference) and Student's unpaired $t$-test. A $P$ value $\leq 0.05$ was considered to be statistically significant.

\section{ACKNOWLEDGMENTS}

We would like to thank Merica Hale and the University of Utah, Department of Dermatology, Dermatopathology Laboratory for adipose tissue histology, and Osama Abdullah and the University of Utah Small Animal Imaging Core MRI for abdominal MRI images. This research was conducted at the University of Utah, Salt Lake City, UT, USA.

\section{STATEMENT OF FINANCIAL SUPPORT}

University of Utah Small Animal Imaging Core MRI instrumentation grant number 1 S10 RR023017.

Disclosure: None of the authors has any potential/perceived conflicts of interest.

\section{REFERENCES}

1. Centers for Disease Control and Prevention. Smoking during pregnancy United States, 1990-2002. MMWR Morb Mortal Wkly Rep 2004;53:911-5.

2. Oken E, Levitan EB, Gillman MW. Maternal smoking during pregnancy and child overweight: systematic review and meta-analysis. Int J Obes (Lond) 2008;32:201-10.

3. Rasmussen EL, Malis C, Jensen CB, et al. Altered fat tissue distribution in young adult men who had low birth weight. Diabetes Care 2005;28:151-3.

4. Ibáñez L, Lopez-Bermejo A, Suárez L, Marcos MV, Díaz M, de Zegher F. Visceral adiposity without overweight in children born small for gestational age. J Clin Endocrinol Metab 2008;93:2079-83.

5. Kim JY, van de Wall E, Laplante M, et al. Obesity-associated improvements in metabolic profile through expansion of adipose tissue. J Clin Invest 2007;117:2621-37.

6. Tran TT, Yamamoto Y, Gesta S, Kahn CR. Beneficial effects of subcutaneous fat transplantation on metabolism. Cell Metab 2008;7:410-20.

7. Chen YC, Chen PC, Hsieh WS, Portnov BA, Chen YA, Lee YL. Environmental factors associated with overweight and obesity in taiwanese children. Paediatr Perinat Epidemiol 2012;26:561-71.

8. Ino T, Shibuya T, Saito K, Inaba Y. Relationship between body mass index of offspring and maternal smoking during pregnancy. Int J Obes (Lond) 2012;36:554-8.

9. Santos-Silva AP, Oliveira E, Pinheiro CR, et al. Endocrine effects of tobacco smoke exposure during lactation in weaned and adult male offspring. J Endocrinol 2013;218:13-24.

10. Gao YJ, Holloway AC, Zeng ZH, et al. Prenatal exposure to nicotine causes postnatal obesity and altered perivascular adipose tissue function. Obes Res 2005;13:687-92.

11. Oliveira E, Moura EG, Santos-Silva AP, et al. Short- and long-term effects of maternal nicotine exposure during lactation on body adiposity, lipid profile, and thyroid function of rat offspring. J Endocrinol 2009;202:397405.

12. Oliveira E, Pinheiro CR, Santos-Silva AP, et al. Nicotine exposure affects mother's and pup's nutritional, biochemical, and hormonal profiles during lactation in rats. J Endocrinol 2010;205:159-70.

13. Hegaard HK, Kjaergaard H, Møller LF, Wachmann H, Ottesen B. The effect of environmental tobacco smoke during pregnancy on birth weight. Acta Obstet Gynecol Scand 2006;85:675-81.

14. Anderson NH, Sadler LC, Stewart AW, Fyfe EM, McCowan LM. Independent risk factors for infants who are small for gestational age by customised birthweight centiles in a multi-ethnic New Zealand population. Aust N Z J Obstet Gynaecol 2013;53:136-42.

15. Bassi JA, Rosso P, Moessinger AC, Blanc WA, James LS. Fetal growth retardation due to maternal tobacco smoke exposure in the rat. Pediatr Res 1984;18:127-30.

16. Ernst M, Moolchan ET, Robinson ML. Behavioral and neural consequences of prenatal exposure to nicotine. J Am Acad Child Adolesc Psychiatry 2001;40:630-41.

17. von Kries R, Bolte G, Baghi L, Toschke AM. Parental smoking and childhood obesity--is maternal smoking in pregnancy the critical exposure? Int J Epidemiol 2008;37:210-6.

18. Janjua NZ, Mahmood B, Islam MA, Goldenberg RL. Maternal and Early Childhood Risk Factors for Overweight and Obesity among Low-Income 
Predominantly Black Children at Age Five Years: a prospective cohort study. J Obes 2012;2012:457173.

19. Nobili V, Alisi A, Panera N, Agostoni C. Low birth weight and catch-upgrowth associated with metabolic syndrome: a ten year systematic review. Pediatr Endocrinol Rev 2008;6:241-7.

20. Jones AP, Simson EL, Friedman MI. Gestational undernutrition and the development of obesity in rats. J Nutr 1984;114:1484-92.

21. Desai M, Gayle D, Babu J, Ross MG. The timing of nutrient restriction during rat pregnancy/lactation alters metabolic syndrome phenotype. Am J Obstet Gynecol 2007;196:555.e1-7.

22. Al Mamun A, Lawlor DA, Alati R, O'Callaghan MJ, Williams GM, Najman JM. Does maternal smoking during pregnancy have a direct effect on future offspring obesity? Evidence from a prospective birth cohort study. Am J Epidemiol 2006;164:317-25.

23. Lacy RT, Hord LL, Morgan AJ, Harrod SB. Intravenous gestational nicotine exposure results in increased motivation for sucrose reward in adult rat offspring. Drug Alcohol Depend 2012;124:299-306.

24. Wake DJ, Rask E, Livingstone DE, Söderberg S, Olsson T, Walker BR. Local and systemic impact of transcriptional up-regulation of 11beta-hydroxysteroid dehydrogenase type 1 in adipose tissue in human obesity. J Clin Endocrinol Metab 2003;88:3983-8.

25. Koska J, de Courten B, Wake DJ, et al. 11beta-hydroxysteroid dehydrogenase type 1 in adipose tissue and prospective changes in body weight and insulin resistance. Obesity (Silver Spring) 2006;14:1515-22.

26. Livingstone DE, Jones GC, Smith K, et al. Understanding the role of glucocorticoids in obesity: tissue-specific alterations of corticosterone metabolism in obese Zucker rats. Endocrinology 2000;141:560-3.

27. Masuzaki H, Paterson J, Shinyama H, et al. A transgenic model of visceral obesity and the metabolic syndrome. Science 2001;294:2166-70.

28. Kotelevtsev Y, Holmes MC, Burchell A, et al. 11beta-hydroxysteroid dehydrogenase type 1 knockout mice show attenuated glucocorticoid-inducible responses and resist hyperglycemia on obesity or stress. Proc Natl Acad Sci U S A 1997;94:14924-9.

29. Nyirenda MJ, Carter R, Tang JI, et al. Prenatal programming of metabolic syndrome in the common marmoset is associated with increased expression of 11beta-hydroxysteroid dehydrogenase type 1. Diabetes 2009;58:2873-9.
30. Pinheiro CR, Oliveira E, Trevenzoli IH, et al. Developmental plasticity in adrenal function and leptin production primed by nicotine exposure during lactation: gender differences in rats. Horm Metab Res 2011;43: 693-701.

31. Boullu-Ciocca S, Achard V, Tassistro V, Dutour A, Grino M. Postnatal programming of glucocorticoid metabolism in rats modulates high-fat dietinduced regulation of visceral adipose tissue glucocorticoid exposure and sensitivity and adiponectin and proinflammatory adipokines gene expression in adulthood. Diabetes 2008;57:669-77.

32. Enzi G, Gasparo M, Biondetti PR, Fiore D, Semisa M, Zurlo F. Subcutaneous and visceral fat distribution according to sex, age, and overweight, evaluated by computed tomography. Am J Clin Nutr 1986;44: $739-46$.

33. Owens S, Gutin B, Ferguson M, Allison J, Karp W, Le NA. Visceral adipose tissue and cardiovascular risk factors in obese children. J Pediatr 1998;133:41-5.

34. Joss-Moore LA, Wang Y, Baack ML, et al. IUGR decreases PPAR $\gamma$ and SETD8 Expression in neonatal rat lung and these effects are ameliorated by maternal DHA supplementation. Early Hum Dev 2010;86:785-91.

35. Zinkhan EK, Fu Q, Wang Y, et al. Maternal Hyperglycemia Disrupts Histone 3 Lysine 36 Trimethylation of the IGF-1 Gene. J Nutr Metab 2012;2012:930364.

36. Chen H, Iglesias MA, Caruso V, Morris MJ. Maternal cigarette smoke exposure contributes to glucose intolerance and decreased brain insulin action in mice offspring independent of maternal diet. PLoS One 2011;6:e27260.

37. Chan DL, Sullivan EA. Teenage smoking in pregnancy and birthweight: a population study, 2001-2004. Med J Aust 2008;188:392-6.

38. Thielen A, Klus H, Müller L. Tobacco smoke: unraveling a controversial subject. Exp Toxicol Pathol 2008;60:141-56.

39. Joss-Moore LA, Wang Y, Campbell MS, et al. Uteroplacental insufficiency increases visceral adiposity and visceral adipose PPARgamma2 expression in male rat offspring prior to the onset of obesity. Early Hum Dev 2010;86:179-85.

40. Livak KJ, Schmittgen TD. Analysis of relative gene expression data using real-time quantitative PCR and the 2(-Delta Delta C(T)) Method. Methods 2001;25:402-8. 